\title{
Link vs. Opto-Electronic Device Sleep Mode Approaches in Survivable Green Optical Networks
}

\author{
Jordi Perelló ${ }^{1}$, Annalisa Morea ${ }^{2}$, Salvatore Spadaro ${ }^{1}$, Massimo Tornatore ${ }^{3}$ \\ (1) Universitat Politècnica de Catalunya (UPC), Barcelona, Spain; (2)Alcatel-Lucent Bell Labs France; \\ (3) Politecnico di Milano(PoliMi), Milano, Italy; e-mail address: perello@ac.upc.edu
}

\begin{abstract}
Energy savings of link and OE device sleep mode approaches are compared in 1:1 protected optical networks, together with a hybrid solution combining both of them. The proposed hybrid solution can decrease network energy consumption by up to $82 \%$. OCIS codes: (060.4250) Networks; (060.4510) Optical communications.
\end{abstract}

\section{Introduction}

The significant increase of energy costs and the need to reduce $\mathrm{CO}_{2}$ emissions have arisen as economic and ecological drivers for a wide range of research activities towards higher energy efficient communications networks [1]. A proposed strategy to increase the energy efficiency of future optical networks is to power transmission systems following the supported traffic variations. To this end, available works in the literature assume that certain optical systems can be put in a low power consumption mode, usually referred as sleep mode, when they are not in use. In most cases, the selected optical systems to be set in sleep mode are link amplifier sites or Opto-Electronic (OE) devices, namely, transponders (TXPs) and regenerators (REGs). In this paper, we refer to these approaches as link sleep mode (LSM) and OE device sleep mode (OESM), respectively.

Survivability is a key feature of current and future optical networks [2]. Although a large number of protection strategies have been proposed to increase their survivability and minimize service downtimes upon failures, all of them rely on the provisioning of additional resources. Such additional resources for protection typically remain unused in non-failure network scenarios, thus becoming candidates to be set in sleep mode under normal network operation. Nevertheless, sleep mode can only be applied to protection resources provided that wake-up times are fast enough to guarantee still acceptable recovery times in case of failure. In this work, we analyze and compare the performance of LSM and OESM approaches in a survivable optical network with dedicated 1:1 protection. We compare the energy consumption reduction that both approaches can achieve in this scenario, while accounting for the limitations of LSM and OESM when managing the power state of the protection resources, given the maximum admissible recovery time imposed by the supported traffic. Particularly, illustrative results are sought for static and reconfigurable optical layer network environments.

\section{Applicability of link and OE device sleep mode approaches in survivable optical networks}

The LSM approach aims at switching off optical amplifiers on unused links in the network to save energy [3]. This strategy is based on the assumption that optical amplifiers can be put in sleep mode, from which they can be rapidly woken-up, for instance, when a new connection has to be provisioned. Nevertheless, no technology has been proposed to this end so far. Furthermore, the dynamic power management of optical amplifiers (e.g., to fast and dynamically change their power state) is currently unsupported by network control plane technologies like GMPLS. Conversely, the OESM approach switches off unused OE devices in the network to save energy. In support of this, the work in [4] proposes a power-manageable TXP/REG architecture implementing three different functional power states: up (fully operational), down (completely unpowered) and idle. In the idle state, the TXP/REG is nonoperational but remains semi-powered, keeping on the components requiring thermal stabilization in order to ensure fast wake-up time in tens of milliseconds [5]. Besides, GMPLS protocol extensions to dynamically manage the power state of OE devices are detailed in [4][5].

In a survivable network scenario, additional resources are allocated for protecting connections upon failures. While remaining unused most of the time, such resources must be rapidly available when a failure arises in order to ensure fast traffic switchover operations. As TXPs/REGs managed by OESM can implement the aforementioned idle state, from which they can be woken-up in a few tens of milliseconds, protection (i.e., backup) resources can be set in idle state under OESM, saving energy when they are inactive. Regarding amplifier sites, however, even if they could be woken-up in tens/hundreds of milliseconds, long-haul links easily comprise many of them that must have to be sequentially activated. This would result in total link wake-up times of seconds, i.e., prohibitive for recovery. Hence, even network links supporting only backup connections must remain in up state the whole time.

Existing works on this topic concentrate either on the power management of amplifiers or TXPs/REGs, while, to the best of our knowledge, no work combines both features. It can be expected, however, that a joint operation of LSM and OESM together should maximally decrease the power consumption of future optical networks. With this 
in mind, we also propose in this work a novel Hybrid Sleep Mode (HSM) approach combining both solutions, which also gives us a benchmark on how far LSM and OESM are behaving with respect to the optimal energy savings.

\section{MILP formulation for quantifying the network energy consumption}

This section presents the MILP formulation that we use to quantify the network energy consumption under LSM, OESM and HSM approaches. The well-known Connectivity Graph (CG) technique has been employed for the translucent optical network design [6], fixing a transparent reach of the optical signals to $1200 \mathrm{Km}$, as suggested for $100 \mathrm{Gbps}$ coherent PDM-QPSK in [7]. For the power consumption of the network elements, we assume that 100Gbps TXPs-REGs consume 350-420W in up state, whereas their consumption falls to 18-36W in idle state [4]. Regarding the bidirectional optical amplifier sites, we assume that they consume $290 \mathrm{~W}$, based on Alcatel-Lucent products [8][9]. These sites contain two EDFA modules in a shelf (one per direction), monitoring system, power supply and fans. A bidirectional amplifier box can only be switched off if not operating in any direction.

Input parameters: $\quad-n^{k},(n)$ : number of REGs at node $n \epsilon N$ supporting lightpaths in - $G(N, E)$ : Physical network topology; $N$ : Set of physical nodes; $\quad$ mode $k$ for the peak-traffic.

$E$ : Set of unidirectional physical fiber links.

- $W$ : Number of wavelengths per physical link.

- $G^{\prime}\left(N, E^{\prime}\right)$ : Network CG; $E^{\prime}$ : Set of logical links.

- $P$ : Set of feasible paths over $G(N, E)$ without regeneration, given the maximum transparent reach of the signal.

- $P_{e^{\prime}}$ : Set of feasible paths over $G(N, E)$ for traffic over $e^{\prime} \epsilon E^{\prime}$.

- $D$ : Set of 1:1 protected demands offered to the network.

- $K$ : Set of lightpath modes, $K=\{$ working, backup $\}$.

- $E_{t}^{k}$ : Energy cons. of a TXP supporting a lightpath in mode $k$.

- $E_{r}^{k}$ : Energy cons. of a REG supporting a lightpath in mode $k$.

- $E_{e}$ : Energy cons. of link e $\epsilon E ; E_{e}=0.5 \cdot\left[\right.$ ceil $\left.\left(D_{e} / 80-1\right)+2\right] \cdot E_{a}$, where $D_{e}$ : length of link $e \in E$ (in km), $E_{a}$ : energy cons. of a bidirectional amplifier box.

- $z_{e} \epsilon_{\{}\{0,1\}: 1$ if $e \in E$ used for the peak-traffic; 0 otherwise.

- $\pi_{\mathrm{pw}} \epsilon\{0,1\}: 1$ if $w \in\{1 . . W\}$ used in $p \in P$ for the peak-traffic; 0 otherwise.

- $C$ : A large constant; $\varepsilon$ : A very small constant.

Decision variables:

- $x^{\mathrm{k}}{ }_{\mathrm{de}}, \epsilon\{0,1\}: 1$ if a lightpath in mode $k \in K$ of $d \in D$ is routed on logical link $e^{\prime} \in E^{\prime} ; 0$ otherwise.

$-y_{d p w}^{k} \epsilon\{0,1\}: 1$ if a lightpath in mode $k \in K$ of $d \epsilon D$ supported on path $p \in P$ with wavelength $w \in\{1 . . W\} ; 0$ otherwise.

- $\pi_{\mathrm{pw}} \epsilon\{0,1\}: 1$ if wavelength $w$ used in path $\mathrm{p} ; 0$ otherwise.

- $z_{e} \epsilon\{0,1\}: 1$ if link $e$ supports at least 1 lightpath; 0 otherwise.

- $n_{t}^{k}(n)$ : num. of TXPs used by lightpaths in mode $k$ at node $n$.

$-n_{r}{ }^{k}(n)$ : num. of REGs used by lightpaths in mode $k$ at node $n$.

Notation:

- $n_{t}^{k}(n)$ : number of TXPs at node $n \in N$ supporting lightpaths in $-a(d)-b(d)$ : source-destination nodes of demand $d \epsilon D$. mode $k$ for the peak-traffic.

$-\langle e>$ : link $e \in E$ or the one in the reverse direction.

Objective function:

$$
\min \sum_{n} \sum_{k}\left(E_{t}^{k} n_{t}^{k}(n)+E_{r}^{k} n_{r}^{k}(n)\right)+\sum_{e} E_{e} z_{e}+\varepsilon \sum_{p} \sum_{w} w \pi_{p w}
$$

Constraints:

$$
\begin{aligned}
\sum_{e^{\prime} \in \mathcal{S}^{+}(n)} x_{d e^{\prime}}^{k}-\sum_{e^{\prime} \in \delta^{-}(n)} x_{d e^{\prime}}^{k}=\left\{\begin{array}{c}
1 \text { if } n=a(d) \\
-1 \text { if } n=b(d) \quad \forall d \in D, k \in K, n \in N . \\
0 \text { otherwise }
\end{array}\right. \\
\sum_{p \in P_{e^{\prime}}} \sum_{w} y_{d p w}^{k}=x_{d e^{\prime}}^{k} \quad e^{\prime} \in E^{\prime}, d \in D, k \in K . \\
\sum_{d} \sum_{p \in P: e \in p} \sum_{k} y_{d p w}^{k} \leq 1 \quad \forall e \in E, w \in\{1, . ., W\} . \\
\sum_{p \in P: e \in p} \sum_{w} \sum_{k} y_{d p w}^{k} \leq 1 \quad \forall d \in D, e \in E . \\
\sum_{d} \sum_{p \in P:\langle e\rangle \in p} \sum_{w} \sum_{k} y_{d p w}^{k} \leq C z_{e} \quad \forall e \in E . \\
\sum_{d \in=a(d) v n=b(d)} 1 \leq n_{t}^{k}(n) \quad \forall n \in N, k \in K . \\
\sum_{e^{\prime} \in \delta^{-}(n)} \sum_{d \in D: n \neq b(d)} x_{d e^{\prime}}^{k} \leq n_{r}^{k}(n) \quad \forall n \in N, k \in K . \\
\sum_{d} \sum_{k} y_{d p w}^{k} \leq \pi_{p w} \quad \forall p \in P, w \in\{1, . ., W\} .
\end{aligned}
$$

Additional constraints for LSM:

$$
\begin{array}{ll}
n_{t}^{k}=n_{t}^{k}{ }^{\prime}(n) & \forall n \in N, k \in K . \\
n_{r}^{k}=n_{r}^{k}{ }^{\prime}(n) & \forall n \in N, k \in K .
\end{array}
$$

Additional constraints for OESM:

$$
\begin{gathered}
\sum_{k} n_{t}^{k}(n) \leq \sum_{k} n_{t}^{k \prime}(n) \quad \forall n \in N . \\
\sum_{k} n_{r}^{k}(n) \leq \sum_{k} n_{r}^{k \prime}(n) \quad \forall n \in N . \\
z_{e}=z_{e}{ }^{\prime} \quad \forall e \in E .
\end{gathered}
$$

Additional constraints for HSM:

$$
\begin{aligned}
& \sum_{k} n_{t}^{k}(n) \leq \sum_{k} n_{t}^{k \prime}(n) \quad \forall n \in N . \\
& \sum_{k} n_{r}^{k}(n) \leq \sum_{k} n_{r}^{k \prime}(n) \quad \forall n \in N .
\end{aligned}
$$

Additional constraints for static optical layer:

$$
\pi_{p w} \leq \pi_{p w}{ }^{\prime} \quad \forall p \in P, w \in\{1, . ., W\} .
$$

Objective function (1) with constraints (2)-(9) are used to optimally design a green translucent optical network satisfying the peak-traffic load. The third term in (1) together with constraints (9) is used to store path-wavelength utilization and foster model convergence. Constraints (2)-(3) ensure that demands are all served. Constraints (4) are the wavelength clashing constraints. Constraints (5) ensure link disjointness between working- backup lightpaths for each demand. Constraints (6)-(9) are used to account for link, TXP, REG and path-wavelength utilization. Then, for 
low-traffic periods, the network is re-designed by including some of the constraints from (10)-(17), depending on the evaluated energy-aware approach and particular optical layer capabilities. Specifically, constraints (17) ensure that for the static optical layer case, the lightpaths in low-traffic periods must be a subset of those established for the peak traffic scenario. This restriction does not exist when the optical layer is reconfigurable.

Table I. Network energy consumption (in kW) achieved by LSM, OESM and HSM approaches

\begin{tabular}{|c|c|c|c|c|c|c|c|c|c|c|c|c|c|}
\hline \multirow{2}{*}{\multicolumn{2}{|c|}{ Scenario }} & \multicolumn{6}{|c|}{ STATIC OPTICAL LAYER CASE } & \multicolumn{6}{|c|}{ RECONFIGURABLE OPTICAL LAYER CASE } \\
\hline & & \multicolumn{2}{|c|}{ Link sleep mode } & \multicolumn{2}{|c|}{ OE device sleep mode } & \multicolumn{2}{|c|}{ Hybrid sleep mode } & \multicolumn{2}{|c|}{ Link sleep mode } & \multicolumn{2}{|c|}{ OE device sleep mode } & \multicolumn{2}{|c|}{ Hybrid sleep mode } \\
\hline & Load [\%] & P(AMP-TXP-REG) & Total & P(AMP-TXP-REG) & Total & P(AMP-TXP-REG) & Total & P(AMP-TXP-REG) & Total & P(AMP-TXP-REG) & Total & P(AMP-TXP-REG) & Total \\
\hline \multirow{5}{*}{$\begin{array}{c}16 \text { Node } \\
\text { EON }\end{array}$} & 100 & $49.88-117.6-58.8$ & 226.28 & $47.27-61.82-20.76$ & 129.85 & |47.27-61.82-20.76 & 129.85 & $49.88-117.6-58.8$ & 226.28 & $47.27-61.82-20.76$ & 129.85 & $\mid 47.27-61.82-20.76$ & 129.85 \\
\hline & 70 & $47.53-117.6-58.8$ & 223.93 & $47.27-43.42-14.47$ & 105.16 & 47.20-43.42-14.57| & 105.19 & $42.79-117.6-58.8$ & 219.19 & $47.27-43.42-14.17$ & 104.86 & $\mid 42.50-43.42-16.47$ & 102.39 \\
\hline & 50 & $45.28-117.6-58.8$ & 221.68 & $47.27-30.91-10.42$ & 88.6 & |43.92-30.91-11.75 & 86.58 & $40.89-117.6-58.8$ & 217.29 & $47.27-30.91-10.36$ & 88.54 & 40.34-30.91-12.22 & 83.47 \\
\hline & 30 & $40.9-117.6-58.8$ & 217.3 & $47.27-18.40-6.27$ & 71.94 & $39.11-18.40-8.46$ & 65.97 & $37.40-117.6-58.8$ & 213.81 & $47.27-18.40-6.00$ & 71.67 & $37.67-18.4$ & 64.79 \\
\hline & 10 & 33.35-117.6-58.8 & 209.75 & 47.27-5.89-1.92 & 55.08 & 31.04-5.89-3.32 & 40.25 & 29.70-117.6-58.8 & 206.1 & 47.27-5.89-1.90 & 55.06 & $30.41-5.8$ & 39.36 \\
\hline \multirow{5}{*}{$\begin{array}{c}\text { COST } \\
239\end{array}$} & 100 & 47.27 & 178.17 & $46.98-58.88-5.88$ & 1111.74 & $46.98-58.88-6.37$ & 112.23 & $12-18.9$ & 178.17 & $46.98-5$ & 111.74 & $46.98-58.8$ & 112.23 \\
\hline & 70 & $46.56-112-18.9$ & 177.46 & $46.98-41.22-4.06$ & 92.26 & $45.95-41.22-4.31$ & 91.475 & $38.95-112-18.9$ & 169.85 & $46.98-41.22-4.01$ & 92.21 & $37.75-41.22-4.67$ & 83.64 \\
\hline & 50 & 42.09-112-18.9 & 172.99 & $46.98-29.44-2.79$ & 79.21 & $40.54-29.44-3.53$ & 73.509 & $32.68-112-18.9$ & $\mid 163.58$ & $46.98-29.44-2.76$ & 79.18 & $\mid 32.19-29.44-4.16$ & 65.79 \\
\hline & 30 & $35.08-112-18.9$ & 165.98 & $46.98-17.66-1.83$ & 66.48 & $34.74-17.66-3.07$ & 55.476 & 27.19-112-18.9 & 158.09 & $46.98-17.66-1.81$ & 66.45 & 25.95-17.66-3.44 & 47.06 \\
\hline & 10 & $24.10-112-18.9$ & 155 & $46.98-5.89-0.50$ & 53.37 & $23.10-5.89-1.53$ & 30.52 & $20.3-112.18 .9$ & 151.2 & $46.98-5.89-0.50$ & 53.37 & $20.59-5.88-1.13$ & 27.61 \\
\hline
\end{tabular}

\section{Performance comparison}

We compare the performance of LSM, OESM and HSM in two different Pan-European network topologies, i.e., the 16-Node EON (16 nodes, 23 links, avg. node degree $=2.88$, avg. link length $=487 \mathrm{~km}$ ) and a small COST 239 network ( 11 nodes, 22 links, avg. node degree $=4$, avg. link length $=421 \mathrm{~km}) .16$ and 10 bidirectional wavelengths per link at 100Gbps are assumed in the 16-Node EON and COST 239 networks, respectively. Regarding the traffic characteristics, in the peak-traffic scenario, we generate a set of 1:1 protected demands between randomly chosen source-destination pairs. The size of this set is large enough to fill the network, but without blocking. The same peak demand set is used for all approaches in a given network. For low-traffic periods, we select a \% of the peak traffic demands randomly and disconnect the remainder. We launch 20 independent model executions per low-traffic load, each time with a new random demand selection, and we average the results. Table I shows the energy consumption achieved by LSM, OESM and HSM in both networks under static vs. reconfigurable optical layer.

OESM and HSM yield drastic energy consumption savings compared to LSM even in highly loaded network scenarios by setting backup TXPs/REGs in idle state. For instance, in the 16-Node EON for a 100\% load, OESM and HSM result in $129.85 \mathrm{~kW}$ in front of the $226.28 \mathrm{~kW}$ of LSM (43\% reduction). Towards lower loads, OESM and HSM behave more efficiently than LSM, as switching off unused TXPs/REGs is easier than finding empty physical links to be shutdown, especially in survivable networks implementing protection. Taking the energy consumption of LSM at $100 \%$ load as a reference, OESM and HSM achieve energy savings in low traffic periods up to $75 \%$ and $82 \%$, respectively, whereas LSM merely saves a $15 \%$. Comparing the results for static/reconfigurable optical layer, we see that OESM behaves very close in both scenarios. In the case of LSM and HSM, they perform slightly better with reconfiguration, as active lightpath rerouting helps in freeing links to be switched off. However, this operation needs complex control plane functions that can cause unacceptable traffic disruptions. Interestingly, HSM yields high energy savings even in the static case, where pernicious active traffic disruptions can be eliminated.

As assumed in this work, OESM and HSM approaches set TXPs/REGs supporting backup lightpaths in idle state to save extra energy, which is enabled by the fast idle-up state transition times. To validate this assumption, we also estimate the recovery time $\left(t_{r}\right)$ that can be incurred when using a backup path in idle state in case of failure. For this, we use the following equation: $t_{r}=R T T+t_{\text {idle-up }} *(N R+2)$, where $R T T$ : round-trip time between source-destination of the backup path (propagation delay during the signaling phase), $t_{\text {idle-up }}$ : idle-to-up state transition time for TXPs/REGs; NR the number of regenerators along the backup path. The term 2 accounts for the source and destination backup TXPs that have to be also woken-up. For example, in the 16-Node EON under OESM and load $100 \%$, average backup path length of $1974 \mathrm{~km}$ and 1.32 REGs per backup path on average are observed. Hence, assuming $t_{\text {idle-up }}=20 \mathrm{~ms}$ [5], we can estimate an average $\mathrm{t}_{\mathrm{r}}=76.3 \mathrm{~ms}$. Under the same load and approach but in the COST 239 network, we obtain $1390 \mathrm{~km}$ and 0.375 REGs per backup path on average, thus leading to $\mathrm{t}_{\mathrm{r}}=54.5 \mathrm{~ms}$. Note that these recovery times are completely acceptable, in view of the recommendations in [10].

\section{Acknowledgements}

This paper has been funded by Spanish Science Ministry through the project ELASTIC (TEC2011-27310).

\section{References}

[1]. www.greentouch.org.

[2]. A. Chiu et al., paper NtuC2, OFC 2009.

[3]. I. Cerutti et al., J. Lightw. Technol. 15, 2292, 2011.

[4]. A. Morea et al, paper We.8.K, ECOC2011.

[5]. A. Morea et al., OM3G.6, OFC 2012.

[6]. M. S. Savasini, et al, ONDM'07, pp. 438-447.

[7]. O. Rival et al., paper OTuI4, OFC 2011.

[8]. http://www.ps2netdrivers.net/manual/alcatel-lucent.1830.pss-1/

[9]. http://www.scribd.com/doc/5033940/1626LM-mannual

[10]. ANSI, Technical Report T1.TR.68-2001. 